\title{
Conservative Treatment for Subungal Hematoma with Tuft Fracture
}

\author{
Zohair Al Aseri \\ College of Medicine, King Saud University Medical City, Riyadh, KSA
}

Received: 02 February, 2017; Accepted: 16 February, 2017; Published: 27 February, 2017

*Corresponding author: Zohair Al Aseri, College of Medicine, King Saud University Medical City, Riyadh, KSA, Tel: +966-1-4670544, 4699347, Fax: +966-1-467 2529; E-mail: zalaseri@ksu.edu.sa

\begin{abstract}
Management of simple nail bed lacerations and subungual hematomas has remained somewhat controversial. This article presents a 30-year-old case admitted to emergency department (ED) due to subungual hematoma with tuft fracture. The nail was partially loose, and the nail bed seemed more or the less intact. Rest of hand examination was normal. Plain radiographs showed fracture of the distal phalanx. Decision was made not to remove the nail bed and only to release the pressure by trephine technique under sterile condition. The patient was scheduled for ED follow up appointment after one week. During follow-up no complications were encountered. The nail bed recovered completely and the nail plate seemed to develop without any deformities. Eight months after the ED visit there are no complaints about pain, shape or loss of function during daily activities. Since there are no agreed upon protocol or algorithm for subungual hematoma and distal phalanx fracture, the author believe that conservative treatment is still an option for treating tuft fracture with subungual hematoma.
\end{abstract}

\section{Abbreviations}

\section{ED: Emergency department}

\section{Background}

Subungual hematoma refers to blood that is trapped under the nail after trauma [1]. In case of fingertip injuries the nail bed is damaged in $15-24 \%$ of the cases [2].

The management of simple nail bed lacerations and subungual hematomas has remained somewhat controversial with much debate surrounding the necessity of removing the nail plate for repair of a nail bed laceration versus trephination alone of a large subungual hematoma [3, 4].

This article presents a 30-year-old case admitted to ED with subungal hematoma and tuft fracture.

\section{Case Presentation}

A 30-year-old healthy male presented himself at ED with an injured little finger after blunt trauma sustained during a construction work. Inspection of the fifth digit of the right hand revealed presence of subungual hematoma. The nail was partially loose, and the nail bed seemed more or the less intact. Rest of hand examination was normal. Plain radiographs showed fracture of the distal phalanx. Decision was made not to remove the nail bed and only to release the pressure by trephine technique under sterile condition. The patient was scheduled for ED follow up appointment after one week. During follow-up no complications were encountered. The nail bed recovered completely and the nail plate seemed to develop without any deformities. Eight months after the ED visit there are no complaints about pain, shape or loss of function during daily activities.

\section{Discussion and Conclusion}

Subungual hematoma (blood under the fingernail or toenail), a common childhood injury, is usually caused by a blow to the distal phalanx (e.g., crush in a door jamb, stubbing one's toe). The blow causes bleeding of the nail bed with resultant subungual hematoma formation. Patients complain of throbbing pain and blue-black discoloration under the nail as the hematoma progresses. Pain is relieved immediately for most patients with simple nail trephination.

Treatment of subungual hematomas covering greater than $25-50 \%$ of the nail bed is controversial. It has been suggested that when $>50 \%$ involvement of the nail plate is associated with a fracture of the distal phalanx the fingernail should be detached, the hematoma drained, and the nail lesions should be identified and eventually treated. Fractures of the distal phalanx are often the result of direct impact, or crush injuries. Most frequently, there is a comminuted tuft fracture $[4,5]$. The nail plate should be removed in the presence of a nail bed hematoma more than $50 \%$ in combination with an intact nail and nail edges, but with a fracture or a visible nail bed laceration. There is no agreed upon management on tuft fracture with subungual hematoma, although some expert exist supporting removing the nail and repair the nail bed $[6,7]$.

Since there are no agreed upon protocol or algorithm for subungual hematoma and distal phalanx fracture, the author believe that conservative treatment is still an option for treating tuft fracture with subungual hematoma. 


\section{Acknowledgements}

All contributors for this study are those included in the author.

\section{References}

1. Nanninga GL, de Leur K, van den Boom AL, de Vries MR, van Ginhoven TM. Case report of nail bed injury after blunt trauma; what lies beneath the nail? Int J Surg Case Rep. 2015;15:133-6.

2. Chang J, Vernadakis AJ, McClellan WT. Fingertip injuries. Clin Occup Environ Med. 2006;5(2):413-22, ix.

3. Patel L. Management of simple nail bed lacerations and subungual hematomas in the emergency department. Pediatr Emerg Care. 2014;30(10):742-5.

4. Tos P, Titolo P, Chirila NL, Catalano F, Artiaco S. Surgical treatment of acute fingernail injuries. J Orthop Traumatol. 2012;13(2):57-62.

5. Giddins GE, Hill RA. Late diagnosis and treatment of crush injuries of the fingertip in children. Injury. 1998;29(6):447-450.

6. Mignemi ME, Unruh KP, Lee DH. Controversies in the treatment of nail bed injuries. J Hand Surg Am. 2013;38(7):1427-30.

7. Seaberg DC, Angelos WJ, Paris PM. Treatment of subungual hematomas with nail trephination: a prospective study. Am J Emerg Med. 1991;9(3):209-10. 